8. Structural integrity of the membranes can be assessed by electron microscopy of sectioned or negatively stained preparations, and the levels of contamination by other organelles (cilia, flagella, cell wall fragments, etc.) can be determined. ${ }^{15}$

Failure to satisfy criterion 2 may not necessarily indicate lack of functional integrity in organisms where nonphosphorylating pathways of electron transport play a significant role in the overall electron flux. ${ }^{12}$

${ }^{15}$ D. F. Parsons, this series, Vol. 10 [101].

\title{
[18] Preparation of Neurospora crassa Mitochondria
}

\author{
By Walter Sebald, Walter Neupert, and Hanns Weiss
}

The fungus Neurospora crassa represents a eukaryotic cell with high biosynthetic activities. Cell mass doubles in 2-4 hr during exponential growth, even in simple salt media with sucrose as the sole carbon source. The microorganism forms a mycelium of long hyphae during vegetative growth. The mitochondria can be isolated under relatively gentle conditions since a few breaks in the threadlike hyphae are sufficient to cause the outflow of the organelles. This article describes two methods for the physical disruption of the hyphae: (1) The cells are opened in a grind mill between two rotating corundum disks. This is a continuous and fast procedure and allows large- and small-scale preparations of mitochondria. (2) Hyphae are ground with sand in a mortar and pestle. This procedure can be applied to microscale preparations of mitochondria starting with minute amounts of cells. Other procedures for the isolation of Neurospora mitochondria after the physical disruption or the enzymatic degradation of the cell wall have been described elsewhere. ${ }^{1}$

\section{Cultivation of Cells}

Neurospora crassa wild type is grown at $25^{\circ}$ in Vogel's minimal medium plus $2 \%$ sucrose. $^{2,3}$ The inoculum is $1-2 \times 10^{6}$ conidia per milliliter.

Cultures of $50 \mathrm{ml}$ are shaken in 200-ml Erlenmeyer flasks on a rotatory shaker at about $150 \mathrm{rpm}$ under sterile conditions. Larger cultures, 0.5-8 liters, are aerated in bottles with a central inlet tube under sterile con-

${ }^{1}$ J. W. Greenawalt, D. O. Hall, and O. C. Wallis, this series, Vol. 10 [27].

${ }^{2}$ H. J. Vogel, Microb. Genet. Bull. 13, 42 (1956).

${ }^{3}$ R. H. Davis and F. J. de Serres, this series, Vol. 17A [4]. 
ditions. For large-scale growth of cells, 100 liters of culture medium are inoculated in a plastic trough with 8 liters of a culture that was grown for about $20 \mathrm{hr}$. The cells are aerated through a central inlet tube. It is not necessary to sterilize the trough, the medium, and the air.

The growth period is $14-24 \mathrm{hr}$ for all three types of cultures. Hyphae grow exponentially up to $20-24 \mathrm{hr}$. The cells are harvested by filtration on filter paper or linen. Large amounts of culture medium are centrifuged in a laundry-type spin drier through a bag of linen. The cells are washed with distilled water. The yield of cells is $5-20 \mathrm{~g}$ wet weight per liter culture medium. One gram wet weight corresponds to about $100 \mathrm{mg}$ dry weight or $40-50 \mathrm{mg}$ protein. For special purposes the cells can be frozen and stored at $-20^{\circ}$

\section{Disruption of the Cell Walls}

All operations are performed at $0^{\circ}-4^{\circ}$. The isolation medium consists of $0.25 M$ sucrose, $1 \mathrm{~m} M$ EDTA, and $10 \mathrm{~m} M$ Tris-acetate, pH 7.2.

Grind Mill (Large- and Small-scale Preparation). ${ }^{4}$ The filtered cells are resuspended in $5-10$ volumes $(\mathrm{w} / \mathrm{v})$ of isolation medium by a $60-\mathrm{sec}$ homogenization in a kitchen blender. The suspended cells are disrupted in a grind mill (Fig. 1) between two rotating corundum disks. The cell suspension is poured into a reservoir and is driven by centrifugal force through the grinding wheels. The homogenate is collected in the case and flows through the outlet tube.

Figure 1 shows the section of a grind mill constructed for large-scale preparations. With this model a flow rate of $10-12$ liters $/ \mathrm{hr}$ is obtained at a grinding pressure of $0.1-0.15 \mathrm{kilopound} / \mathrm{cm}^{2}$. For small-scale preparations a mill can be built in which all parts are reduced in size by a factor of about 3 .

Sand and Mortar (Microscale Preparation). The filtered cells, 0.1$0.5 \mathrm{~g}$ wet weight, are mixed with an equal weight of quartz sand (washed and calcinated, reagent grade) in a mortar (inner diameter $4-5 \mathrm{~cm}$ ). One volume $(\mathrm{v} / \mathrm{w})$ of isolation medium is added, and the mixture is ground with a pestle until a smooth paste is obtained (about $2 \mathrm{~min}$ ). Another volume of medium is added, and the grinding is continued for a further 2 min. Finally, the homogenized slurry is transferred with 2 volumes of isolation medium into microcentrifuge tubes of $1.4 \mathrm{ml}$ (Fa. Eppendorff, Hamburg, Germany).

${ }^{4}$ H. Weiss, G. von Jagow, M. Klingenberg, and T. Bücher, Eur. J. Biochem. 14, 75 (1970). 


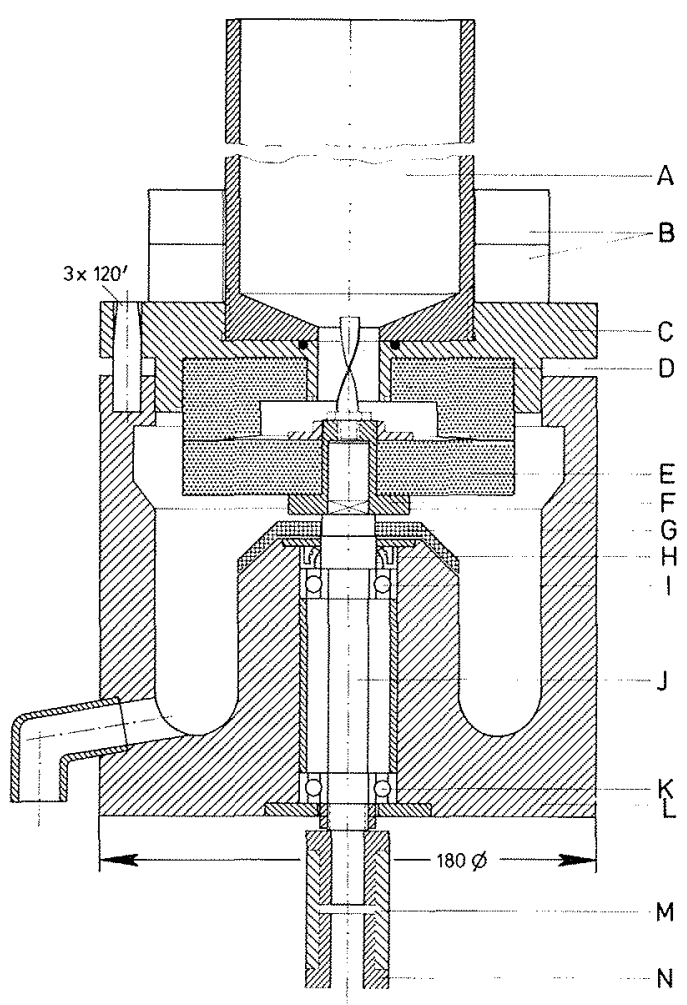

FIG. 1. Section of the grind mill. The upper (D) and lower (E) grinding wheels consist of corundum (Edelkorund 89 A60 M5 V, Fa. Tyrolit, Schwaz, Austria). The upper wheel (D) is glued into the round plastic disk (C) made from hard polyvinyl chloride. The reservoir (A) is a tube of Plexiglas $30 \mathrm{~cm}$ long and is screwed into the disk (C). The lead rings (B) have a weight of $2 \mathrm{~kg}$ each. The holding device for the lower wheel (E) is the screw (F), which fits exactly into the central hole. The screw $(F)$ has on the bottom side a square bore, which fits to the end of the movable shaft $(J)$. On its upper side the screw carries a stirrer. The shaft $(\mathrm{J})$ rotates in the ball bearings $(\mathrm{I}, \mathrm{K})$ and is protected from moisture by the covering $(G)$ and the gasket $(H)$. The case $(L)$ is made from hard polyvinyl chloride. The shaft $(J)$ is connected by means of the flexible coupling $(M)$ to the electric motor $(N)$ not shown in the figure $(1300 \mathrm{rpm}$; at least $150 \mathrm{~W})$. The diameter of the case is $180 \mathrm{~mm}$. The lower grinding wheel is rotated by the electric motor. Its central hole is closed by a holding device. The upper grinding wheel does not rotate and can be lifted. Its central hole is open and connected with the reservoir cylinder. The upper grinding wheel is pressed against the lower wheel by the lead weights. The grinding area is reduced by a conical cut of the lower surface of the upper wheel. 
The efficiency of the disruption procedures can be checked by inspection in a phase-contrast microscope. The empty cells appear dark, whereas the broken cells are bright and show particulate cell contents. ${ }^{4}$

\section{Isolation of Mitochondria}

The mitochondria are isolated from cells disrupted by either of the two methods described above by differential centrifugation between 1200 $g(10 \mathrm{~min})$ and $8000 \mathrm{~g}(30 \mathrm{~min})$. The low-speed sediment is usually completely colorless. The high-speed centrifugation may also be performed at $15,000 \mathrm{~g}$ for $15 \mathrm{~min}$. When "mitochondria" are isolated from frozen cells the time for high-speed centrifugation is doubled. The crude mitochondrial fraction is resuspended in isolation medium (10\% of the starting volume), and the low- and high-speed centrifugations are repeated. For the microscale preparation of mitochondria the microtubes can be used in a preparative refrigerated centrifuge with appropriate adaptors.

The yield of mitochondria is $2-3 \mathrm{mg} / \mathrm{g}$ wet weight of cells. About 25 $\%$ of the total mitochondria are isolated, which corresponds to $4-6 \%$ of total cellular protein. ${ }^{4}$

\section{Properties of Isolated Neurospora Mitochondria}

Respiratory Chain and Oxidative Phosphorylation. The mitochondria isolated by means of the grind mill show a high rate of respiration with pyruvate plus malate, succinate, NADH, and NADPH, which is fairly well coupled to oxidative phosphorylation. Three phosphorylation steps are involved in the oxidation of pyruvate, and two are involved in the oxidation of succinate, NADH, and NADPH. ${ }^{4}$ Rotenone, antimycin, and $\mathrm{KCN}$ inhibit the electron flow at the known steps. Oligomycin, venturicidin, and dicyclohexylcarbodiimide (DCCD) inhibit mitochondrial ATPase half-maximally at $0.2,0.2$, and $1 \mu \mathrm{g} / \mathrm{mg}$ mitochondrial protein, respectively. The molar ratio of cytochromes $a a_{3}, c, c_{1}, b-_{562}$, and $b_{-556}$ is $1.0: 2.9: 1.0: 0.9: 0.9$. The content of cytochrome $a a_{3}$ is $0.24-0.34 \mu \mathrm{mol} /$ mg mitochondrial protein. ${ }^{5}$

In Vitro Protein Synthesis. The mitochondria synthesize proteins at a rate of 5-10 pmol leucine incorporated per minute and milligram mitochondrial protein. ${ }^{6}$ The leucine incorporation is in the same range as that observed with mitochondria isolated after enzymatic degradation of

${ }^{5}$ G. von Jagow, H. Weiss, and M. Klingenberg, Eur. J. Biochem. 33, 140 (1973).

${ }^{6}$ A. von Ruecker, S. Werner, and W. Neupert, FEBS Lett. 47, 290 (1974). 
the cell wall, ${ }^{7}$ but it does not exceed $4-8 \%$ of the incorporation rate observed in vivo in intact cells. The in vitro synthesis of cytochrome oxidase subunits could be demonstrated. ${ }^{6}$

Isolation of Mitochondrial Components. The mitochondria have been used as starting material for the isolation of cytochrome oxidase, ${ }^{8,9} \mathrm{cy}$ tochrome $b{ }^{10,11}$ ATPase complex and $F_{1}$ ATPase, ${ }^{12,13}$ carboxyatractyloside-binding protein, ${ }^{14}$ and ribosomes ${ }^{15}$ and for the separation of outer and inner membrane. ${ }^{16}$ The proteins can be prepared from mitochondria labeled homogeneously with radioactive amino acids. ${ }^{17}$ This offers the advantage that protein is determined by radioactivity measurements.

The cytochromes and $F_{1}$ ATPase can be prepared from cells frozen for several weeks at $-20^{\circ}$. The complete subunit pattern of the $F_{1}$ ATPase is obtained only when mitochondria have been isolated in the presence of the protease inhibitor phenylmethylsulfonyl fluoride (PMSF). The presence of proteinase in the mitochondrial preparation is largely due to the coisolation of proteinase-containing vesicles. ${ }^{18}$ Mitochondria can be freed of the proteinase vesicles by sucrose density gradient centrifugation. "Mitochondria" isolated from frozen cells are largely free of this proteinase, since the proteinase vesicles are disrupted by freeze-thawing. ${ }^{18}$

Immunoprecipitation. For biogenetic studies, ${ }^{17}$ membrane-bound enzyme complexes can be isolated by immunological techniques. Neurospora mitochondria are almost completely dissolved by Triton X-100. From the lysate, cytochrome oxidase, ${ }^{19}$ a free subunit of cytochrome oxidase, ${ }^{19}$ and the ATPase complex ${ }^{12,13}$ have been immunoprecipitated by means of specific antisera. In these studies the microscale preparation of mitochondria has been applied to small amounts of cells highly labeled by in vivo incorporation of radioactive amino acids. ${ }^{17}$

${ }^{7}$ W. Sebald, T. Bücher, B. Olbrich, and F. Kaudewitz, FEBS Lett. 1, 235 (1968).

${ }^{8}$ H. Weiss, W. Sebald, and T. Bücher, Eur. J. Biochem. 22, 19 (1971).

${ }^{9} \mathrm{H}$. Weiss and W. Sebald, this series, Vol. 53 [11].

${ }^{10} \mathrm{H}$. Weiss and B. Ziganke, Eur. J. Biochem. 41, 63 (1974).

${ }^{11} \mathrm{H}$. Weiss and B. Ziganke, this series, Vol. 53 [23].

${ }^{12}$ G. Jackl and W. Sebald, Eur, J. Biochem, 54, 97 (1975).

${ }^{13} \mathrm{~W}$. Sebald and G. Wild, this volume, Article [41].

${ }^{14}$ M. Klingenberg, H. Aquila, P. Ricchio, B. B. Buchanan, W. Eiermann, and H. Hackenberg, in "Electron Transfer Chains and Oxidative Phophorylation" (E. Quagliariello et al., eds.), p. 431. North-Holland Publ., Amsterdam, 1975.

${ }^{15}$ W. Neupert, G. Hallermeyer, and R. Michel, this series, Vol. 56 [8].

${ }^{16}$ W. Neupert and G. D. Ludwig, Eur. J. Biochem. 19, 523 (1971).

${ }^{17}$ W. Sebald, S. Werner, and H. Weiss, this series, Vol. 56 [5].

${ }^{18}$ R. Michel, A. Liebl, A. Hartmann, and W. Neupert, Hoppe-Seyler's Z. Physiol. Chem. 357, 415 (1976).

${ }^{19}$ S. Werner, Eur. J. Biochem. 43, 39 (1974). 